\title{
Child with SARS-CoV-2 infection and acute lymphoblastic leukemia
}

\author{
Raluca Bontea', Geta Vancea ${ }^{1,2}$, Dana Ispas ${ }^{1}$, Nicoleta Tudor', Nicoleta Voicu', \\ Cristina Iordache ${ }^{1}$, Gabriela Scurtu', Andreea Stoenescu ${ }^{1,2}$, Andreea Popica', \\ Claudia Chirila', Simin-Aysel Florescu, ${ }^{1,2}$, Emanoil Ceausu ${ }^{1,2,3}$, Petre Calistru ${ }^{1,2}$ \\ 1 "Dr. Victor Babes" Clinical Hospital of Infectious and Tropical Diseases, Bucharest, Romania \\ 2 "Carol Davila" University of Medicine and Pharmacy, Bucharest, Romania \\ ${ }^{3}$ Academy of Medical Sciences, Romania
}

\begin{abstract}
Introduction. Coronavirus disease 2019 (COVID-19) frequently leads to asymptomatic or mild infectious disease evolution in children.

Case presentation. We present the case of a 3 year old girl, known with acute lymphoblastic leukemia in chemotherapy treatment at that time. She had an asymptomatic form of COVID-19 but with important paraclinical changes. The evolution was favorable under the treatment initiated with antibiotics, corticotherapy, gastric protector and symptomatic treatment if necessary.

Conclusion. Management of children with COVID-19 and other comorbidities remains a challenge given the few data currently in the literature
\end{abstract}

Keywords: COVID-19, acute lymphoblastic leukemia, immunodepression, children

\section{INTRODUCTION}

First appearing in Wuhan, China, in December 2019, SARS-CoV-2 virus infection has spread rapidly to all continents. Children are less affected compared to adults. In children, the signs and symptoms of the disease are comparable to those of adults with a predominance of mild and even asymptomatic forms of the disease $(1,2)$. Unlike other viral pathogens, it appears that SARS-CoV-2 infection does not present a higher risk of respiratory complications in immunosuppressed patients, destruction of lung tissue during infection also depends on the host's immune response (3). Currently, there are few data on the impact of COVID-19 on the immunocompromised population. Most studies focus on adults, with very few studies on immunosuppressed children.

\section{CASE PRESENTATION}

We present the case of a child, female, aged 3 years and 4 months, hospitalized in our clinic in May 2020. The child comes from twin pregnancy and he is known with acute lymphoblastic leukemia type B in clinical and hematological remission (diagnosed in September 2018) undergoing treatment with Methotrexate, Purinetol, Sumetrolim and Liv52. The background treatment was stopped about a week before hospitalization at the indication of the hematologist who observed in the routine tests of the patient leukopenia with neutropenia. Given the epidemiological context at that time, the attending physician recommended to perform the SARS-CoV-2 RT-PCR. The test confirmed the infection with the new coronavirus, which is why she was admitted to our clinic for surveillance, investigations and specialized treatment. 
Informed consent of the mother was obtained for clinical examination, blood tests and treatment of the child.

\section{Clinical findings}

The child did not show any clinical signs or symptoms during admission.

Diagnostic focus and assessment: Biological investigations at the time of presentation showed: leukopenia $(3,000 / \mu \mathrm{l})$, neutropenia $(500 / \mu \mathrm{l})$, lymphopenia $(1,600 / \mu \mathrm{l})$, slightly increased ALT (92 U/1), the rest of the analyzes (inflammation markers, coagulation samples, IL-6, ferritin, creatinine, glucose, ionogram, urine summary, pharyngeal exudate) being within normal limits. Pulmonary radiography shows interstitial infiltrates with minimal associated alveolar component, diffusely distributed in the lower halves of both lung fields.

\section{Therapeutic focus and assessment}

Because the radiological image raises the high suspicion of bacterial infection in a host immunocompromised by the underlying disease and the chemotherapy treatment received, to which was added corticotherapy, it was decided to start the treatment, for curative and prophylactic purposes for possible severe bacterial infection, the patient received treatment with Cefort $1400 \mathrm{mg} /$ day iv is instituted - administered in two doses, for 3 days, and because it could not be maintained venous approach, it was decided to replace it with Augmentin $400 \mathrm{mg} / 57 \mathrm{mg} / 5 \mathrm{ml}, 12 \mathrm{ml} /$ day, administered in two doses, for 7 days, Azithromycin $200 \mathrm{mg} / 5 \mathrm{ml}, 5 \mathrm{ml} /$ day po, for 10 days, Dexamethasone $6 \mathrm{mg} /$ day iv, administered in two doses, for 7 days then $3 \mathrm{mg} /$ day iv, administered in two doses, 2 more days, Omez 10 $\mathrm{mg} /$ day po and Liv52. On the eighth day of hospitalization, the SARS-CoV-2 RT-PCR test was repeated with a negative result. The clinical and paraclinical evolution of the child was good, the radiological and biological investigations returning to normal during the 10-day hospitalization.

\section{REFERENCES}

1. Minotti $C$, Tirelli F, Barbieri E, Giaquinto $C$, Donà $D$. How is immunosuppressive status affecting children and adults in SARS-CoV-2 infection? A systematic review. J Infect. 2020;81(1):e61-e66.

2. Taub JW, Ge Y, Xavier AC. COVID-19 and childhood acute lymphoblastic leukemia. Pediatr Blood Cancer. 2020;67(7):e28400.

3. Marcia M, Vania B, Pruccoli G, Vallero SG, Barisone E, Scolfaro C, Fagioli F. Acute lymphoblastic leukemia onset in a 3-year-old child with COVID-19. Pediatr Blood Cancer. 2020 Nov;67(11):e28423.

4. Razanamahery J, Soumagne T, Humbert S, Brunel AS, Lepiller Q, Daguindau E, Mansi L, Chirouze C, Bouiller K. Does type of immunosupression influence the course of COVID-19 infection? J Infect. 2020 Aug;81(2):e132-e135.

5. Shaunak M, Patel R, Driessens C, et al. COVID-19 symptom surveillance in immunocompromised children and young people in

\section{Follow-up and monitoring}

The child was able to resume the treatment of the underlying disease after discharge. He was monitored by his family doctor and his hematologist specialist.

\section{DISCUSSION}

Immunocompromised child undergoing chemotherapy for acute lymphoblastic leukemia, interrupted treatment 7 days prior to hospitalization. He has an asymptomatic form of COVID-19 but with important paraclinical changes. The response was favorable to the antibiotic and cortisone treatment initiated without receiving Hydroxychloroquine or Lopinavir / Ritonavir recommended in most treatment protocols at that time, the need for this specific treatment being assessed according to the characteristics of each case. Studies show that immunosuppressed patients with COVID-19 have more forms of the disease with favorable evolution compared to patients with other comorbidities (cardiovascular disease, diabetes, obesity) $(2,3,6,7)$. This can be explained by a hypothetical protective role regarding a severe evolution of the disease due to the low immune response in this category of patients $(3,4,5,8)$. However, immunocompromised patients, even if asymptomatic, with mild or moderate disease, require repeated clinical and paraclinical evaluations during SARS-CoV-2 virus infection until healing, to detect in advance a possible unfavorable course of the disease.

\section{CONCLUSIONS}

It is confirmed that in childhood SARS-CoV-2 infection often develops asymptomatically. In our case, the asymptomatic evolution was present in a child with immunosuppression caused by a hematological underlying disease with undergoing chemotherapy with favorable evolution.

Conflict of interest: none declared Financial support: none declared

the UK: a prospective observational cohort study. BMJ Open. 2021;11(3):e044899.

6. Pérez-Martinez A, Guerra-García P, Melgosa M, Frauca E, FernandezCamblor C, Remesal A, Calvo C. Clinical outcome of SARS-CoV-2 infection in immunosuppressed children in Spain. Eur J Pediatr. 2021 Mar;180(3):967-971.

7. Nicastro E, Verdoni L, Bettini LR, Zuin G, Balduzzi A, Montini G, Biondi A, D’Antiga L. COVID-19 in Immunosuppressed Children. Front Pediatr. 2021 Apr 29;9:629240.

8. Meena JP, Kumar Gupta A, Tanwar P, Ram Jat K, Mohan Pandey R, Seth $R$. Clinical presentations and outcomes of children with cancer and COVID-19: A systematic review. Pediatr Blood Cancer. 2021 Jun;68(6):e29005. 